Please do not remove this page

RMIT

UNIVERSITY

\title{
Transparent electrodes based on spray coated fluorine-doped tin oxide with enhanced optical, electrical and mechanical properties
}

Kim, Jaewon; Wong, Sherman; Kim, Gahui; Park, Young-Bae; van Embden, Joel; Della Gaspera, Enrico https://researchrepository.rmit.edu.au/esploro/outputs/9921970140301341/filesAndLinks?institution=61RMIT_INST\&index=null

Kim, J., Wong, S., Kim, G., Park, Y.-B., van Embden, J., \& Della Gaspera, E. (2020). Transparent electrodes based on spray coated fluorine-doped tin oxide with enhanced optical, electrical and mechanical properties. Journal of Materials Chemistry C, 8(41), 14531-14539. https://doi.org/10.1039/d0tc03314f Document Version: Accepted Manuscript

Published Version: https://doi.org/10.1039/d0tc03314f 


\title{
Enhanced Optical, Electrical and Mechanical Properties in Fluorine-Doped Tin Oxide Transparent Electrodes
}

\author{
Jaewon Kimª, Sherman Wong ${ }^{\mathrm{a}}$, Gahui Kim ${ }^{\mathrm{b}}$, Young-Bae Park ${ }^{\mathrm{b}}$, Joel van Embden ${ }^{\mathrm{a}}$, Enrico Della \\ Gaspera $^{\mathrm{a}, *}$
}

\begin{abstract}
Transparent electrodes are key components of optoelectronic devices such as solar cells, light emitting diodes and touchscreen displays. Despite great progress, there remains several challenges to be addressed in transparent conducting coatings, especially for achieving high-performance materials with scalable and inexpensive production. Here we present the deposition of fluorine-doped tin oxide (FTO) thin films with enhanced optoelectronic and mechanical properties using ultrasonic spray pyrolysis. We systematically investigate the influence of deposition temperature and doping amount on the structural, electrical, optical and mechanical properties of FTO films using a suite of characterization techniques including Xray diffraction, scanning electron microscopy, optical spectroscopy, Hall effect measurement and nanoindentation. We discovered that at a given dopant level, grain size dictates the electrical and mechanical properties, with larger grain sizes and the reduction in grain boundaries - achieved at higher deposition temperatures - promoting an increase in electron mobility and in mechanical strength due to the inverse Hall-Petch effect. The effect of fluorine doping is complementary to that of deposition temperature: the amount of fluorine does not significantly affect grain size and mechanical properties, however it has a great effect on the optical and electrical properties of FTO. Using optimized deposition conditions we achieved FTO coatings with excellent optoelectronic properties (electrical resistivity $\sim 7 \times 10^{-4} \Omega \cdot \mathrm{cm}$; visible transparency $\sim 90$ $\%)$ and enhanced mechanical properties (elastic modulus $\sim 170 \mathrm{GPa}$; hardness $\sim 16 \mathrm{GPa}$ ). These results are some of the best ever reported for FTO films, and provide a solid base for their direct implementation within optoelectronic devices.
\end{abstract}

\section{Introduction}

Modern electronics have seen a rise in the implementation of touch functionalities, with touchscreen displays now being one of their fundamental components. This, in conjunction with other widespread applications including photovoltaics, LED displays and "smart" (dimmable, energy efficient) windows, has generated a steady increase in the production of inexpensive and efficient transparent electrodes. Transparent electrodes are thin coatings that provide electrical contact to a device, while enabling light (mostly in the visible range) to be transmitted. As such, these are the components that enable solar radiation to reach the light-absorbing layer in photovoltaic devices - and at the same time to extract the photogenerated charges - as well as light to be emitted from LED displays including TVs, smartphones and tablets. ${ }^{1,2}$

The most common choice for transparent electrode applications is a class of materials known as transparent conductive oxides (TCOs) due to the combination of optical transparency, electrical conductivity and chemical stability they provide. Among all TCOs, tin dioxide, $\mathrm{SnO}_{2}$, is a prime candidate due to its strong $n$-type character and its large fundamental band gap ( $3.6 \mathrm{eV}$ for bulk, undoped $\mathrm{SnO}_{2}$ ) which permits complete visible transparency. In addition, $\mathrm{SnO}_{2}$ presents distinctive advantages over other TCO materials (e.g. indium tin oxide, ITO), including low-toxicity, high thermal and environmental stability, high mechanical durability and low-cost due to the relative abundance of tin on the Earth's crust. ${ }^{3}$ The native high conductivity of pure $\mathrm{SnO}_{2}$ can be ascribed to the presence of intrinsic defects, such as oxygen vacancies, and can be drastically enhanced through extrinsic aliovalent dopants, which include both cations (such as antimony and niobium) and anions (such as fluorine). Coatings of tin oxide, especially when doped with fluorine (FTO), have achieved conductivities greater than $10^{5} \mathrm{~S} / \mathrm{m}$, with optical transparency values in the visible range above $80 \%$, making them one of the main industry standards for transparent electrode applications. ${ }^{1,4-8}$

The structural, optical and electrical properties of doped $\mathrm{SnO}_{2}$ have been extensively studied as a function of the experimental parameters for both vacuum-based and solution-based depositions. Top performing coatings can achieve excellent electrical (sheet resistance <20 $\Omega / \square$ ) and optical (visible transparency exceeding $85 \%$ ) properties. 4, 6, 7, 9-13,14 Mechanical properties such as elastic modulus and hardness also play an important role in ensuring the reliability of these coatings when utilized within devices. However, only a few selected works have studied the mechanical properties of doped $\mathrm{SnO}_{2}$ thin films. ${ }^{15-17}$ For example, it has been reported that the deposition of doped $\mathrm{SnO}_{2}$ using wet chemical methods produces coatings with lower hardness ( $<10 \mathrm{GPa}$ ) compared to films deposited via vacuum-based methods such as sputtering ( $\mathrm{H} \sim 15-20 \mathrm{GPa}$ ), although the values reported in the literature are fairly inconsistent. ${ }^{17,18,19}$ The origin of this lower hardness is typically ascribed to the presence of structural and morphological defects such as pores and grain boundaries which are prevalent in coatings obtained from solution-based depositions.

In this work, we present a detailed study on the properties of FTO coatings deposited by ultrasonic spray pyrolysis. Spray pyrolysis has been utilized for decades for the deposition of oxide coatings from precursors solutions, because it enables fast, reliable and conformal depositions with great operational versatility and potentially low waste. ${ }^{20}$ When an ultrasonic atomizer is adopted, coatings of exceptional quality can be achieved, in terms of both uniformity and thickness control. ${ }^{21,22}$ We analyze and discuss the effect of key experimental conditions (deposition temperature and dopant concentration) on the structural, electrical, optical and mechanical properties of the deposited FTO coatings. We identify an optimal window 
in the parameter space that enables the deposition of highly transparent (85-90\% transmittance), highly conductive FTO coatings (conductivity $>10^{5} \mathrm{~S} / \mathrm{m}$ ) that also possess enhanced mechanical properties (hardness $\sim 15 \mathrm{GPa}$, elastic modulus > $150 \mathrm{GPa}$ ) compared to all other currently available FTO coatings deposited from liquid precursors.

\section{Experimental section}

\section{Materials}

Tin chloride pentahydrate $\left(\mathrm{SnCl}_{4} \cdot 5 \mathrm{H}_{2} \mathrm{O}, 98 \%\right)$ and ammonium fluoride $\left(\mathrm{NH}_{4} \mathrm{~F},>99.99 \%\right)$ were purchased from Sigma-Aldrich. Ethanol (99.5\%), isopropanol (IPA, 99.5\%) and acetone (99.5\%) were obtained from Univar. All chemicals were used without further purification.

\section{FTO thin film fabrication}

Fluorine doped $\mathrm{SnO}_{2}$ thin films were deposited via ultrasonic spray pyrolysis of sol-gel precursors. $\mathrm{SnCl}_{4} \cdot 5 \mathrm{H}_{2} \mathrm{O}$ as the tin precursor and $\mathrm{NH}_{4} \mathrm{~F}$ as the dopant precursor were dissolved in ethanol and the overall concentration $(\mathrm{S} n+\mathrm{F})$ was kept constant at $0.2 \mathrm{M}$. Commercial borosilicate glass substrates were cleaned ultrasonically in acetone and IPA for 10 minutes each, then dried with a stream of nitrogen. The ultrasonic spray system used for FTO films deposition (ND-SP spray coater, Nadetech innovations) consists of a $40 \mathrm{kHz}$ ultrasonic spray nozzle mounted on a gantry enabling software-controlled $X-Y$ movements, an air compressor to control the shape of the spray mist and a syringe pump to deliver the precursor solution at a constant feed rate. Borosilicate substrates were positioned on a hotplate and then heated to the desired temperature and let stabilize for 10 minutes prior to deposition. The spray coating parameters were optimized separately and kept constant throughout this study. In details, the distance between nozzle and substrate was set at $105 \mathrm{~mm}$; the solution flow rate at 1 $\mathrm{mL} / \mathrm{min}$; the speed of the spray head at $10 \mathrm{~mm} / \mathrm{sec}$; the air pressure at 0.5 bar; finally the coatings were achieved by depositing multiple layers through subsequent passes of the spray head (30 layers for each sample). The main parameters varied in this study were the deposition temperature $\left(380^{\circ} \mathrm{C}\right.$ to $460^{\circ} \mathrm{C}$ ) and the amount of fluorine dopant ( 0 to $40 \%$ nominal). The nominal fluorine concentration $X$ in percent is defined as:

$$
X=\frac{n_{F}}{n_{F}+n_{S n}} \times 100
$$

where $n$ is the number of moles. The samples have been labelled FTOX accordingly. After the deposition was completed, the samples were kept for an additional 15 minutes at the same temperature, and then cooled slowly to room temperature to prevent thermal shock.

\section{Characterization techniques}

X-ray diffraction (XRD) scans of the FTO films were obtained with a Bruker D4 diffractometer operating at $40 \mathrm{kV}$ and $35 \mathrm{~mA}$ using Cu K $\alpha$ radiation (1.5418 ̊). The Scherrer relationship was used to calculate the crystallite size after obtaining the full width at half maximum (FWHM) of the diffraction peaks fitted with Lorentzian functions. The preferred orientation of the crystalline grains was evaluated through analysis of the texture coefficient $P$ obtained by the following formula:23,24

$$
P\left(h_{i} k_{i} l_{i}\right)=\frac{I\left(h_{i} k_{i} l_{i}\right)}{I_{0}\left(h_{i} k_{i} l_{i}\right)}\left[\frac{1}{n} \sum_{i=1}^{n} \frac{I\left(h_{i} k_{i} l_{i}\right)}{I_{0}\left(h_{i} k_{i} l_{i}\right)}\right]^{-1}
$$

where $I_{0}$ is the predicted intensity for randomly oriented crystals (obtained from the ICDD cards), $l$ is the experimental intensity of the $\left(h_{i}, k_{i}, l_{i}\right)$ diffraction and $n$ is the number of peaks taken into consideration. The surface morphology and thickness of FTO films were measured by field emission scanning electron microscopy (FE-SEM, FEI Nova NanoSEM). The electrical properties (carrier concentration, mobility and resistivity) were measured using an Ecopia HMS-3000 Hall effect system at room temperature in van der Pauw geometry. Optical transmission spectra were acquired with an Agilent Cary 5000 spectrophotometer. X-ray photoelectron spectroscopy (XPS) was conducted with a Kratos AXIS Supra X-ray photoelectron spectrometer equipped with a monochromated Al Ka X-ray source $(1486.7 \mathrm{eV})$. Mechanical properties such as elastic modulus $(E)$ and hardness $(H)$ of FTO coatings were obtained by nanoindentation (Hysitron TI-950 triboindenter). The indenter tip was loaded at least 9 times per sample, each time in a new spot at least $7.5 \mu \mathrm{m}$ apart from the previous. The loaddisplacement curves were measured with a loading time of 10 seconds, a hold time of 5 seconds, and an unloading time of 10 seconds. To assess the effect of the penetration depth, dedicated tests were performed with maximum penetration ranging from $10 \mathrm{~nm}$ to $250 \mathrm{~nm}$. In order to confirm that the tip shape did not affect the measured mechanical properties, two different tips were used: a pyramidal Berkovich tip with a nominal radius of $40 \mathrm{~nm}$ and a cono-spherical tip with a radius of $5 \mu \mathrm{m}$. The elastic modulus and hardness were evaluated from load-unload curves corresponding to $\max 15 \%$ penetration depth into the thin film surface, and then an average value plus/minus the standard deviation was presented.

\section{Results and discussion}

\section{FTO thin film deposition}

As described in the experimental procedure, fluorine-doped tin oxide was deposited on borosilicate glass substrates using ethanolic solutions of the precursors. Ethanol was chosen as a solvent because of its low toxicity, good ability to dissolve the tin and fluorine precursors and excellent wetting for glass substrates, enabling high-quality films. To further improve the quality of the coatings and achieve the desired thickness, the FTO films were deposited through multiple layers or "passes" of the spray head as schematically presented in Figure 1a. With this method, dense FTO thin films of high quality can be prepared, as demonstrated in the digital photograph and in key characterizations presented in Figure 1. 

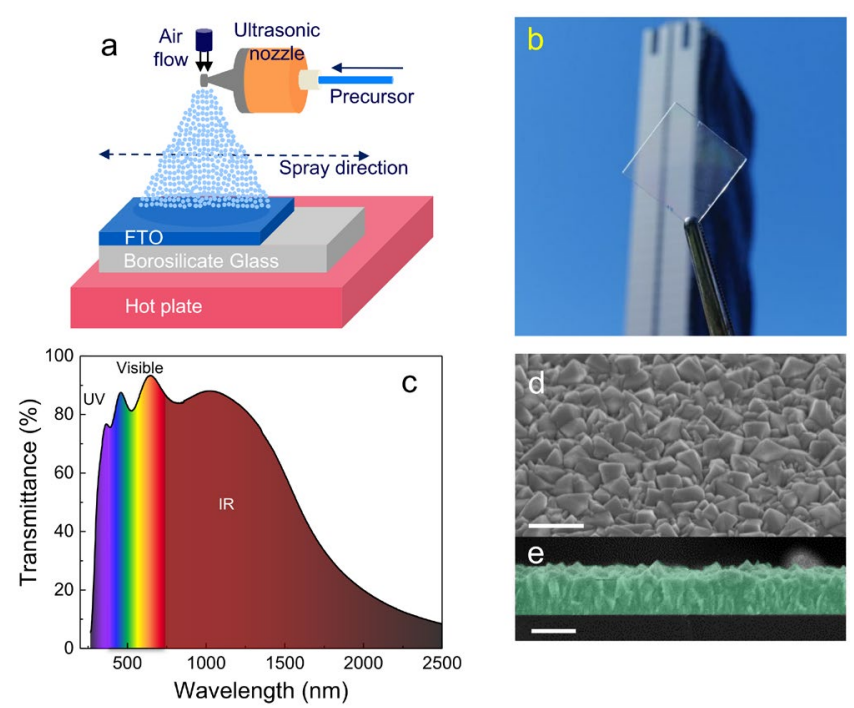

Fig. 1 Fluorine doped $\mathrm{SnO}_{2}$ deposited by ultrasonic spray pyrolysis. (a) Schematic of the ultrasonic spray pyrolysis system. (b) A photograph of FTO thin film on glass. (c) Optica transmittance spectrum of a typical FTO thin film. (d,e) SEM images of FTO film in top tilted view (d) and cross section (e, false-colored). Scale bars are $500 \mathrm{~nm}$.

\section{Structural Properties}

X-ray diffraction (XRD) was used to analyze the crystallinity and the crystal orientation of the deposited films. The results obtained for FTO20 samples deposited at various temperatures are presented in Figure 2 and Figure S1 in the ESI. The diffraction patterns of all samples deposited above $400{ }^{\circ} \mathrm{C}$ can be assigned to the tetragonal rutile phase of $\mathrm{SnO}_{2}$ (cassiterite, ICDD No. 00041-1445), while samples deposited at lower temperatures are mostly amorphous or poorly crystalline. Moreover, samples deposited at $440{ }^{\circ} \mathrm{C}$ and above show sharper diffraction peaks with higher intensity, suggesting larger grains and increased crystallinity, as expected from thermally-induced crystallization and grain growth. Interestingly, a couple of minor peaks are observed in samples deposited at $400{ }^{\circ} \mathrm{C}$ and $420{ }^{\circ} \mathrm{C}$, possibly due to orthorhombic $\mathrm{SnO}_{2}$ or tetragonal $\mathrm{SnO}$, which are not detected in coatings deposited at higher temperatures.
Therefore, highly crystalline and phase pure $\mathrm{SnO}_{2}$ can be obtained at $440{ }^{\circ} \mathrm{C}$.

The crystallite size estimated from the full width at half maximum (FWHM) of the main diffraction peaks as a function of the deposition temperature is shown in Figure $2 \mathrm{~b}$. As expected, higher deposition temperatures trigger the formation of larger crystalline grains, with the crystallite size roughly doubling when increasing the deposition temperature from 400 ${ }^{\circ} \mathrm{C}$ to $460{ }^{\circ} \mathrm{C}$ (from $13.5 \pm 8.0 \mathrm{~nm}$ to $27.8 \pm 5.0 \mathrm{~nm}$, respectively). It should be noted that the Scherrer formula used to estimate crystallite size is rather qualitative and provides a rough estimation, and as such it is useful mostly for identifying trends rather than providing precise numbers. Importantly, samples deposited at the same temperature but with different amount of fluorine doping show minimal differences in crystallite size, except a slight increase at low doping levels as shown in Figure S2.
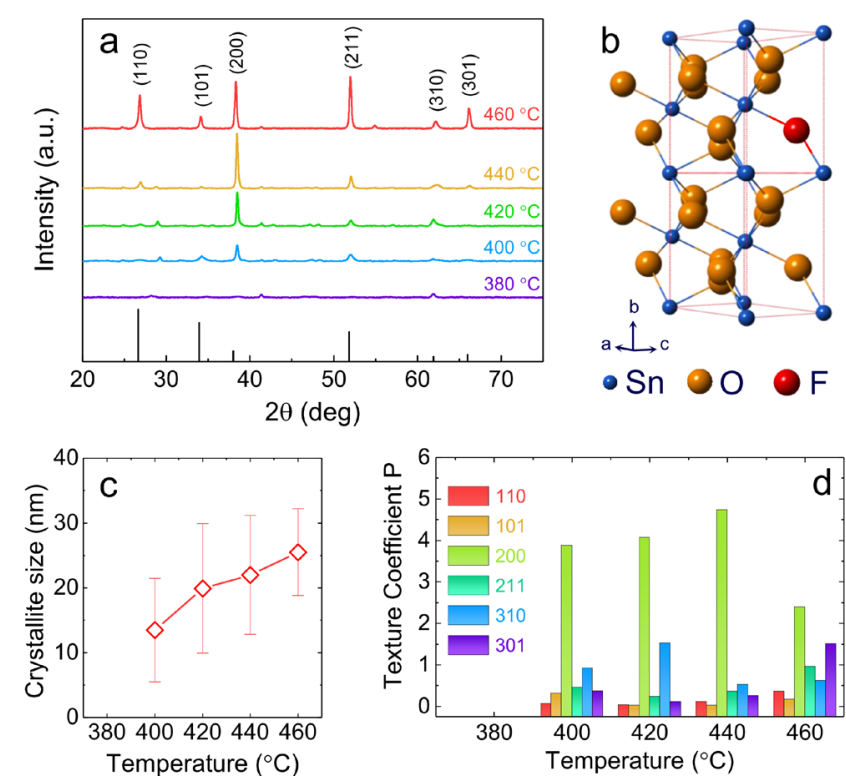

Fig. 2 a) XRD patterns for FTO20 films deposited at various deposition temperatures. The expected peak positions for rutile $\mathrm{SnO}_{2}$ according to ICDD No. 00-041-1445 are reported at the bottom. b) Schematic representation of the FTO lattice. c) Crystallite size calculated from the diffraction patterns presented in panel (a). d) Texture coefficient for FTO20 as function of deposition temperature.

The orientation of the crystals is also found to depend on the amount of fluorine doping. The texture along the (200) direction slowly decreases with increasing the amount of 
fluorine up to $30 \%$, and then drastically reduces to almost unoriented crystals for larger dopant amounts, suggesting that major changes in the crystal structure happen when a large amount of aliovalent dopant is introduced (Figure S3). Interestingly, the position of the diffraction peaks is almost unaffected by the incorporation of fluorine dopants (the largest peak shifts are of the order of $0.05^{\circ}$, corresponding to variation in lattice parameters $<0.15 \%$ ), suggesting minimal perturbation in cell size upon fluorine doping.

\section{Surface Morphology}

The surface morphology of FTO thin films was analyzed by SEM and the results are reported in Figure 3a-e.
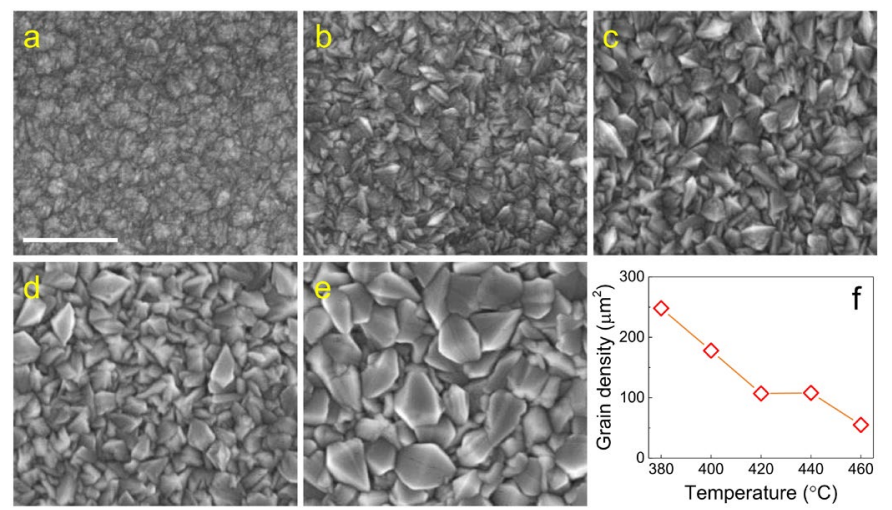

The morphology was found to be heavily dependent on the growth temperature. At low deposition temperatures $\left(380^{\circ} \mathrm{C}\right)$ the crystalline grains are very small and hard to detect, which is consistent with the amorphous nature of the films as confirmed by XRD. At $400{ }^{\circ} \mathrm{C}$ and above, the films show larger, more faceted grains which are also seen to further increase in size with deposition temperature. The areal density of the crystalline grains plotted as a function of the deposition temperature shows a constant decrease in the number of grains per unit area with increasing temperature (Figure 3f). These conclusions are further corroborated by the cross-sectional SEM images shown in Figure S4. Similarly to its effect on crystallite size, the amount of fluorine does not significantly influence the morphology of the coatings deposited at the same temperature, as shown in Figure S5.

\section{Electrical and optical properties}

Having analyzed the morphological and structural properties of FTO films, we now move to their electrical properties. Figure 4a shows the carrier concentration, mobility and resistivity of the FTO thin films as a function of the deposition temperature acquired using Hall effect measurements performed at room temperature. All the measured films displayed a negative Hall coefficient, which is indicative of n-type conductivity, as expected for $\mathrm{SnO}_{2}$-based materials.

The carrier concentration in samples prepared with constant fluorine loading is seen to increase with deposition temperature up to $420{ }^{\circ} \mathrm{C}$ (from $1.1 \times 10^{20} \mathrm{~cm}^{-3}$ to $4.6 \times 10^{20} \mathrm{~cm}^{-3}$ ), and then decreases for films deposited at higher temperatures (down to $2.5 \times 10^{20} \mathrm{~cm}^{-3}$ for $460{ }^{\circ} \mathrm{C}$ ). The increase in carrier concentration from $380^{\circ} \mathrm{C}$ to $420^{\circ} \mathrm{C}$ implies that progressively more fluorine is successfully incorporated within the tin oxide lattice, and/or that a larger fraction of dopant is active and therefore contributes to the generation of free carriers. The decrease observed at higher temperatures may be related to a combination of the solubility limit of fluorine dopant in the tin oxide lattice, the increase disorder due to additional dopant being incorporated, or the volatilisation of byproducts containing fluorine before dopant atoms could be actually incorporated within the growing $\mathrm{SnO}_{2}$ crystals. ${ }^{10}$

Unsurprisingly, the electron mobility increases steadily with deposition temperature, with a 4-fold increase from $6.6 \mathrm{~cm}^{2} / \mathrm{V} \cdot \mathrm{s}$ to $27.0 \mathrm{~cm}^{2} / \mathrm{V} \cdot \mathrm{s}$ for samples deposited between $380^{\circ} \mathrm{C}$ and 460 ${ }^{\circ} \mathrm{C}$. This enhanced mobility is in good agreement with the increased grain size and reduced grain boundaries (and therefore the associated charge scattering) observed by SEM. The net effect of these two contributions on the electrical resistivity is a sharp decrease in resistivity for deposition temperatures up to $420^{\circ} \mathrm{C}$, after which the resistivity plateaus to a value of $\sim 9 \times 10^{-4} \Omega \cdot \mathrm{cm}$.

Another parameter that greatly influences the electrical property of FTO is the amount of fluorine doping. This is clearly shown in the Hall effect results presented in Figure S6. The carrier concentration increases $\sim 4$-fold from undoped $\mathrm{SnO}_{2}(<1$ $\left.\times 10^{20} \mathrm{~cm}^{-3}\right)$ to highly doped FTO $\left(\sim 4.2 \times 10^{20} \mathrm{~cm}^{-3}\right)$, even if the carrier concentration almost plateaus for fluorine concentrations $>\mathbf{2 0} \%$. In general, the mobility slowly decreases with fluorine doping, in agreement with aliovalent dopants causing stresses and defects within the lattice. In fact, a combination of ionised impurity scattering, and dopant selfcompensation (i.e. formation of acceptor defects such as interstitial fluorine that partially counterbalance the effect of substitutional doping) has been found to be responsible for reduced mobility in highly doped $\mathrm{SnO}_{2}{ }^{26}$ Interestingly, small amounts of fluorine cause an increase in mobility compared to undoped $\mathrm{SnO}_{2}$. The combination of the trends in carrier concentration and electron mobilty produces a sharp decrease in resistivity for low fluorine doping levels, and a negligible effect on electrical resistivity for fluorine amounts $>20 \%$. We also conducted electrical measurements at low temperatures on a FTO20 sample and observed a positive temperature coefficient of resistance $\left(\sim 8.5 \times 10^{-3} \mathrm{~K}^{-1}\right.$ between $300 \mathrm{~K}$ and 200 $\mathrm{K}$, and $\sim 2.9 \times 10^{-3} \mathrm{~K}^{-1}$ between $200 \mathrm{~K}$ and $20 \mathrm{~K}$ ), which is indicative of metallic-like conductivity, confirming the excellent electrical properties of these FTO coatings (Figure S7).

In addition to their electrical conductivity, the other main property that needs to be maximized in transparent electrodes is their optical transmission in the visible spectral range. The transmittance spectra of FTO20 samples deposited at various pyrolysis temperatures are presented in Figure $4 \mathrm{~b}$. An overall increase in the average transmittance in the visible range with increasing deposition temperature is observed. Specifically, the average transmittance increases from $\sim 77 \%$ to over $86 \%$ when the deposition temperature is raised from $380^{\circ} \mathrm{C}$ to $440{ }^{\circ} \mathrm{C}$. At higher deposition temperatures, the transmittance is seen to decrease. The low transmittance at deposition temperatures below $400{ }^{\circ} \mathrm{C}$ is believed to be due to the incomplete decomposition and volatilization of the tin precursor during the 
spray pyrolysis process. The reduction in transmittance observed in samples deposited at $460{ }^{\circ} \mathrm{C}$ is due to their larger grain size and rougher surface, which causes non-negligible scattering of light. These considerations are well supported by XRD and SEM results discussed earlier. All samples show a marked decrease in transmittance in the near infrared, associated with the surface plasmon resonance triggered by free carriers. The intensity of this IR absorption matches very well the carrier concentration evaluated via Hall effect, with the

sample deposited at $420{ }^{\circ} \mathrm{C}$ showing the most blue-shifted onset. In fact, the frequency of the plasmon resonance is directly related to the number of free carriers, and therefore samples with higher carrier concentration should show a sharper decrease in transmittance at lower wavelengths.

The amount of fluorine dopant does not significantly affect the overall visible transparency of the films, which remains > $85 \%$ for all fluorine concentrations evaluated in this study. However, the dopant presence strongly affects the amount of infrared absorption, in agreement with the increased free electron concentration as shown in Figure S8. The increased doping level is also confirmed by the widening of the optical band gap as a function of the amount of fluorine used in the precursor solution. A progressively stronger Burstein-Moss shift is observed at high fluorine doping, providing additional confirmation of the generation of free carriers (Figure S9). The conductivity of our FTO films fabricated with the optimal experimental parameters $\left(440{ }^{\circ} \mathrm{C}\right.$ deposition temperature and $20 \%$ amount of fluorine) is $>1 \times 10^{5} \mathrm{~S} / \mathrm{m}$, which coupled to their excellent optical transmittance (average $91.3 \%$ in the $400-800$ $\mathrm{nm}$ range, with peak transmittance of $\sim 93 \%$ at $550 \mathrm{~nm}$ ) provides an excellent combination of properties for application as transparent electrodes. By also increasing the film thickness, we could achieve even better electrical properties (sheet resistance $10.6 \Omega / \square$ ) without affecting the optical transparency (average transmittance: $90.7 \%$, with peak transmittance at $550 \mathrm{~nm}$ of $93.9 \%)$, resulting in a value for the Haake figure of merit of over $35 \mathrm{~m}^{-1}$ (which increases to $\sim 50 \mathrm{~m}^{-1}$ if using the peak transmittance). ${ }^{27}$ This value is one of the best reported for FTO films, and is on par with or better than commercial FTO products. ${ }^{7,10,25,28-30}$ Key parameters evaluated from the optical and electrical measurements for FTO samples prepared in this study are summarized in Table S1.
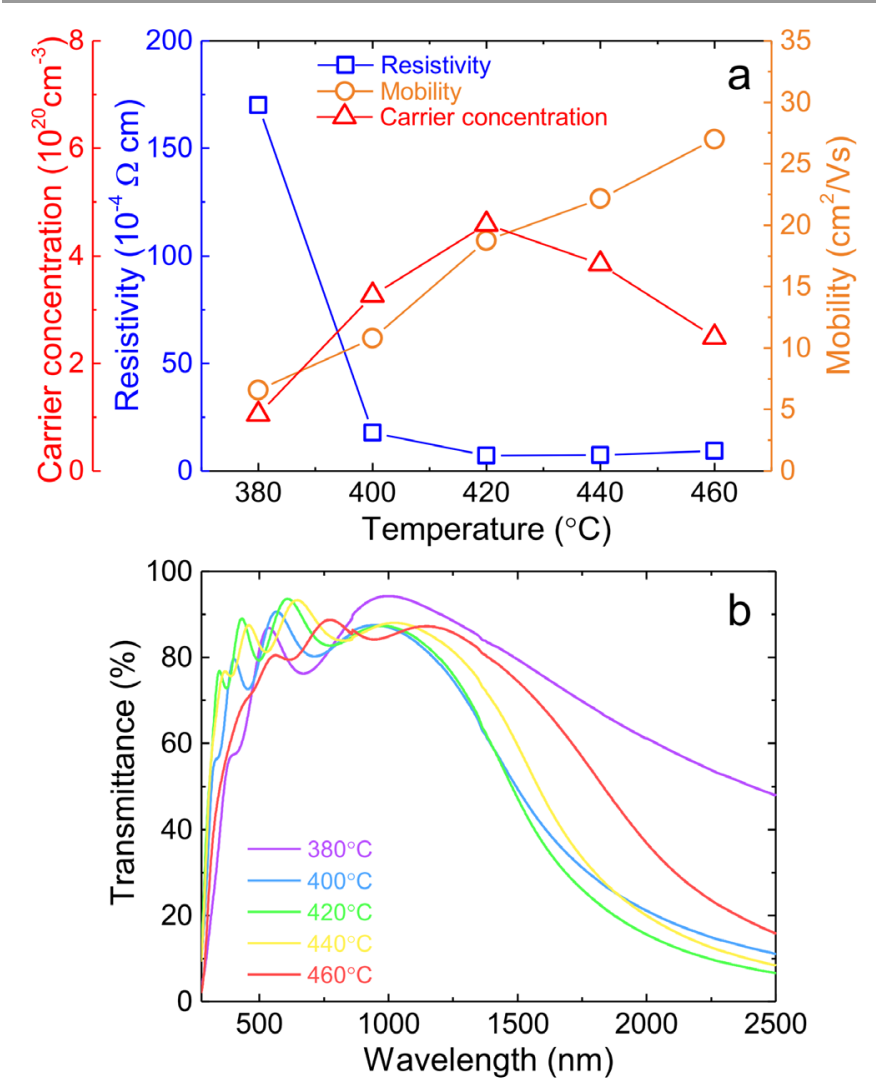

Fig. 4 Electrical and optical properties of FTO films deposited at different deposition temperatures. a) Carrier concentration, mobility and resistivity evaluated via Hall effect measurements. b) Optical transmittance spectra.

The chemical composition and the electronic properties of undoped and doped $\mathrm{SnO}_{2}$ films were probed via XPS and the results are presented in Figure S10. A clear peak centered at about $686.5 \mathrm{eV}$ is observed in FTO samples, confirming the presence of fluorine, while undoped samples do not display any signal in the $\mathrm{F} 1 \mathrm{~s}$ spectral region, as expected. The $\mathrm{F}$ 1s peak is slightly asymmetric, and can be fitted with two separate components, one at lower binding energies ascribed to substitutional fluorine at the oxygen site, and one at higher binding energies due to interstitial fluorine. The latter constitutes an acceptor defect, which partially counterbalances the donors provided by substitutional doping. The ratio between the interstitial and substitutional components is 0.51 , in agreement with previous results, suggesting that for every two fluorine atoms acting as donors, there is one fluorine acting as acceptor. ${ }^{26}$ The tin core level spectra, and especially the Sn $4 \mathrm{~d}$ peaks, show a clear change in line shape with fluorine doping, which is ascribed to a progressive separation between the screened and unscreened component, and again due to the plasmon resonance and the presence of free carriers. A thorough description of the model can be found in the literature. ${ }^{9,}{ }^{31}$ Additional confirmation of the metallic nature of our FTO is obtained analysing the valence band spectra: besides three distinctive peaks typically observed in $\mathrm{SnO}_{2}$ and ascribed to $S n 5 s, S n 5 d$ and $O 2 p$ orbitals, doped samples display a stronger contribution at the Fermi energy, ascribed to the photoemission of electrons located within the conduction band, and therefore indicative of the presence of metallic states. ${ }^{4,32,33}$ 


\section{Mechanical Properties}

Understanding the role of morphology and interfaces on the mechanical properties is of crucial importance when fabricating multi-layer devices such as optoelectronics, and therefore the analysis of the mechanical properties of FTO thin films is vital, and yet surprisingly overlooked. We used nanoindentation in a variety of configurations to probe the mechanical behavior of FTO, and extracted hardness and elastic modulus values for samples deposited at different temperatures and different doping levels. This technique relies on the progressive penetration of a tip of known shape into the specimen, and both the applied force and the penetration depth are recorded during the loading and unloading cycles. When performing nanoindentation measurements on coatings, there are a few considerations that need to be taken into account to obtain reliable results. It has been previously shown that the mechanical properties of coatings are related to the indentation depth. ${ }^{34}$ However, when performing nanoindentation measurements on very thin films (thinner than a few micrometers), the mechanical properties are significantly influenced by those of the substrate. Therefore, it has been suggested in the literature that the indentation depth should be limited to $10-15 \%$ of the total thin film thickness to minimise the substrate contribution, especially in thin coatings, which is the case for our FTO films. ${ }^{35}$

Figure 5a shows a typical load-displacement curve obtained via nanoindentation for a FTO20 film deposited on borosilicate glass at $440{ }^{\circ} \mathrm{C}$. When the tip is driven into the surface of the material, the sample undergoes both elastic and plastic deformation. Due to the plastic deformation, after one complete cycle, the displacement (depth) at zero loading is nonzero. The elastic modulus $(E)$ and the hardness $(H)$ can be calculated using the Oliver and Pharr method from the loaddisplacement data through the following equations: ${ }^{36}$

$$
\begin{gathered}
E=\frac{\sqrt{\pi}}{2} \frac{1}{\sqrt{A_{\max }}} \frac{\Delta P}{\Delta h} \\
H=\frac{P_{\max }}{A_{\max }}
\end{gathered}
$$

where $\Delta P / \Delta h$ is the slope of the indentation unloading curve at the maximum applied load, $P_{\max }$ is the maximum applied load and $A_{\max }$ is the projected contact area under $P_{\text {max }}$.

Figure $5 \mathrm{~b}$ presents the elastic modulus and hardness for a typical FTO20 thin film as a function of the maximum indentation depth using either a Berkovich probe or a conospherical probe. For both tip shapes, an initial decrease in elastic modulus as the indentation tip penetrated though the thin film was observed ( $1^{\text {st }}$ stage), while the value of the measured elastic modulus plateaued beyond $60 \mathrm{~nm}$ depth $\left(2^{\text {nd }}\right.$ stage). This behaviour is typical of thin films and can be explained in the following way: the first stage at low penetration depths is due to the intrinsic elastic modulus of the contacted film, and in this region the substrate contribution is minimized. The roughly constant or slightly decreasing values obtained at higher penetration depths (second stage) is consistent with the mechanical properties being mostly affected by the presence of the substrate. ${ }^{16,37,38}$
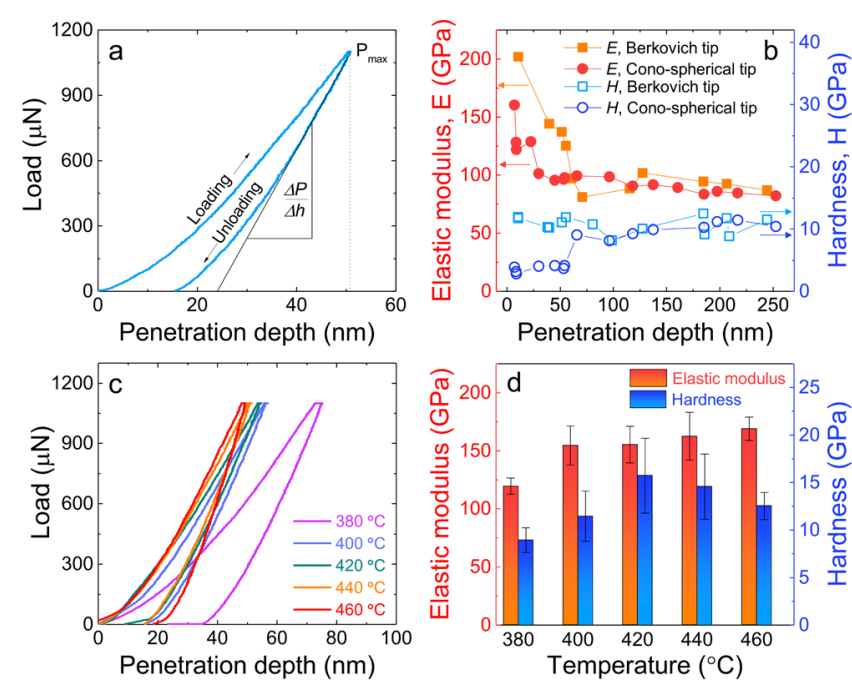

Fig. 5 Nanomechanical characterization of FTO films. a) Typical nanoindentation load-displacement curve showing a single load/unload cycle. b) Elastic modulus and hardness as a function of the penetration depth evaluated using Berkovich tip and cono-spherical tips for the same FTO20 sample. c) Load-displacement curves as a function of deposition temperature. d) Elastic modulus and hardness of FTO20 films deposited at various temperatures. 
Table 1: Elastic modulus $(E)$ and hardness $(H)$ for $\mathrm{SnO}_{2}$-based thin films fabricated in this study and for other $\mathrm{SnO}_{2}$-based oxides reported in the literature. Some of the key deposition parameters are also listed. $39,40,15,16,17$

\begin{tabular}{|c|c|c|c|c|c|c|}
\hline Sample & Technique & Temperature $\left({ }^{\circ} \mathrm{C}\right)$ & Thickness (nm) & $E(\mathrm{GPa})$ & $H(\mathrm{GPa})$ & Reference \\
\hline ATO & CVD & 425 & $\mathrm{~N} / \mathrm{A}$ & 73 & 5.9 & 39 \\
\hline ATO & Spray pyrolysis & 550 & N/A & 33 & 3.4 & 39 \\
\hline ATO & Spin coating & 600 & 150 & 46.8 & 4.34 & 40 \\
\hline $\mathrm{Cd}_{2} \mathrm{SnO}_{4}$ & Dip-coating & 550 & 250 & 82.1 & 4.9 & 15 \\
\hline FTO & Spray pyrolysis & 400 & 297 & 131.7 & 12.3 & 16 \\
\hline FTO & CVD & 600 & $\mathrm{~N} / \mathrm{A}$ & 75.1 & 9.9 & 17 \\
\hline \multirow{3}{*}{ FTO } & \multirow{3}{*}{ Spray pyrolysis } & 400 & 302 & 154.5 & 11.4 & \multirow{3}{*}{ This work } \\
\hline & & 440 & 344 & 162.5 & 14.6 & \\
\hline & & 460 & 405 & 168.8 & 12.5 & \\
\hline
\end{tabular}

Typical load-displacement curves for FTO20 samples deposited at various deposition temperatures are shown in Figure $5 \mathrm{c}$. For these measurements, a maximum load of $1100 \mu \mathrm{N}$ was applied in all samples. It can be clearly seen that using the same applied load, the tip penetration is progressively reduced in samples deposited at higher temperatures, and this is particularly evident analyzing the sample deposited at $380{ }^{\circ} \mathrm{C}$. This trend is presented in Figure S11 and suggests that more rigid films are obtained at higher deposition temperatures. The mechanical properties (elastic modulus and hardness) obtained from the load-displacement curves for samples deposited at different temperatures are shown in Figure $5 \mathrm{~d}$. The elastic modulus is clearly dependent on the temperature, showing a progressive increase with increased pyrolysis temperature, especially between $380{ }^{\circ} \mathrm{C}$ and $400{ }^{\circ} \mathrm{C}$. The main morphological and structural differences that were observed as a function of the deposition temperature are the crystallinity of the samples and the size of the grains. Therefore, we can ascribe the improved mechanical properties measured at high deposition temperatures to the increased crystallinity and increased grain size (reduced grain density). This is in good agreement with the inverse Hall-Petch effect observed in nanoscale materials, which is governed by grain boundary sliding. ${ }^{16,41}$ The hardness is also seen to slightly increase with deposition temperature, which is consistent with the increased crystallinity. However, the hardness does not increase anymore for temperatures above $420^{\circ} \mathrm{C}$, and on the contrary, a reduction in hardness is seen at $460{ }^{\circ} \mathrm{C}$, which could be ascribed with the loss of preferential orientation, and potentially to hardness being dependant on the crystallographic directions as observed in other inorganic materials. ${ }^{42}$

While deposition temperature strongly affects the mechanical properties of FTO, the concentration of fluorine does not contribute to substantial changes as shown in Figure S12. This evidence matches reasonably well the SEM results and the grain size analysis, which showed no significant differences in sample prepared with different fluorine levels. However, at the highest doping levels the mechanical properties started to deteriorate, most likely as a consequence of defects and local disorder caused by excess fluorine within the $\mathrm{SnO}_{2}$ lattice. These results suggest that low concentrations of fluorine do not affect significantly the crystallinity and the defectivity of $\mathrm{SnO}_{2}$, providing mechanical properties comparable to undoped $\mathrm{SnO}_{2}$, but at the same time generating the desired optical and electrical properties in FTO films which are suitable for applications as transparent electrodes. Finally, another important parameter is the adhesion of FTO coatings onto the glass substrates. Both undoped $\mathrm{SnO}_{2}$ and FTO films show excellent adhesion to the borosilicate substrate as demonstrated via 4-point bending tests (see Figure S13). ${ }^{43,44}$ In these measurements, a crack is generated on the substrate until its tip is near the bonding interface, and then the crack is propagated further via a bending stress. A weak interface would cause the crack to change direction when reaching said interface, causing delamination. In all our tests, the crack propagation penetrated through the $\mathrm{SnO}_{2}$ and FTO films instead of through the glass/film interface. For this reason, the actual quantification of the interfacial toughness is not possible. However, the lack of delamination in any of the performed tests suggests strong adhesion between film and substrate. The detailed description of these experiments is reported in the ESI following Figure S13. A summary of the mechanical properties evaluated for some of the key samples prepared in this study, along with the comparison with other tin-based oxide materials is reported in Table 1. Our samples are on par, or better than previously reported results for solution-processed $\mathrm{SnO}_{2}$-based coatings, further confirming the high quality of our FTO films not just for their optoelectronic properties, but also for their mechanical properties.

\section{Conclusion}

In conclusion, we have presented a detailed investigation into the deposition of polycrystalline fluorine-doped tin oxide (FTO) films via ultrasonic spray pyrolysis. The use of an ultrasonic atomizer and precise control of substrate temperature enables the fabrication of dense, conformal and crystalline tin oxide coatings using simple and inexpensive liquid precursors. We demonstrated that the deposition conditions strongly influence the structural, optical, electrical and mechanical properties of FTO films. In details, higher pyrolysis temperatures favor the formation of large crystalline grains, which enhance the electron mobility (through a reduced number of grain boundaries) and mechanical properties (through the inverse 
Hall-Petch effect). In contrast, the concentration of fluorine dopants did not significantly modify the film structure (crystallinity, grain size) or mechanical properties (hardness, elastic modulus). However, the presence of fluorine does exert a strong effect on the optical and electrical properties, promoting tunable infrared absorption, and enhanced conductivity exceeding $10^{5} \mathrm{~S} / \mathrm{m}$, while still maintaining excellent visible transparency (>85\%, with peak transmittance $\sim 90 \%$ ).

This systematic work proves that spray pyrolyzed FTO can exhibit superior properties compared to existing conventional methods and establishes solution-processed FTO coatings as a competitive technology for the implementation of TCOs within high performance optoelectronic devices.

\section{Conflicts of interest}

Authors declare no conflicts of interest.

\section{Acknowledgements}

The Australian Research Council is acknowledged for supporting this work through the following grants: DE170100164 (EDG), DP190101864 (EDG), DE150100427 (JVE). The authors acknowledge the facilities and the technical assistance of the RMIT University's Microscopy and Microanalysis Facility (RMMF). Dr James Partridge is acknowledged for his help with Hall effect measurements.

\section{References}

1. K. Ellmer, Nature Photonics, 2012, 6, 809-817.

2. M. Morales-Masis, S. De Wolf, R. Woods-Robinson, J. W. Ager and C. Ballif, Advanced Electronic Materials, 2017, 3, 1600529.

3. D. S. Ginley, H. Hosono and D. C. Paine, Handbook of transparent conductors, Springer, 2010.

4. S. D. Ponja, B. A. D. Williamson, S. Sathasivam, D. O. Scanlon, I. P. Parkin and C. J. Carmalt, Journal of Materials Chemistry C, 2018, 6, 7257-7266.

5. J. P. Correa Baena and A. G. Agrios, ACS Appl Mater Interfaces, 2014, 6, 19127-19134.

6. G. Rey, C. Ternon, M. Modreanu, X. Mescot, V. Consonni and D. Bellet, Journal of Applied Physics, 2013, 114, 183713.

7. A. Muthukumar, G. Rey, G. Giusti, V. Consonni, E. Appert, H. Roussel, A. Dakshnamoorthy and D. Bellet, AIP Conference Proceedings, 2013, 1512, 710-711.

8. J. Yang, B. Liang, M. Zhao, Y. Gao, F. Zhang and H. Zhao, Scientific reports, 2015, 5, 15001.

9. J. I. Scott, R. F. Martinez-Gazoni, M. W. Allen and R. J. Reeves, Journal of Applied Physics, 2019, 126, 135702.

10. O. S. Elsherif, G. E. A. Muftah, O. Abubaker and I. M. Dharmadasa, Journal of Materials Science: Materials in Electronics, 2016, 27, 12280-12286.

11. V. Consonni, G. Rey, H. Roussel and D. Bellet, Journal of Applied Physics, 2012, 111, 033523.

12. N. M. Nguyen, M. Q. Luu, M. H. Nguyen, D. T. Nguyen, T. T. Truong and T. Nguyen-Tran, Journal of Electronic Materials, 2017, 46, 3667-3673.
13. R. Ramarajan, M. Kovendhan, K. Thangaraju, D. P. Joseph and R. R. Babu, Applied Surface Science, 2019, 487, 13851393.

14. J. Kim, B. Murdoch, J. Partridge, K. Xing, D.-C. Qi, J. LiptonDuffin, C. F. McConville, J. van Embden and E. Della Gaspera, Advanced Materials Interfaces, 2020, DOI: 10.1002/admi.202000655.

15. C. J. Diliegros-Godines, F. J. Flores-Ruiz, R. CastanedoPérez, G. Torres-Delgado, F. J. Espinoza-Beltrán and E. Broitman, Journal of Sol-Gel Science and Technology, 2014, 74, 114-120.

16. L. T. C. Tuyen, S. R. Jian, N. T. Tien and P. H. Le, Materials (Basel), 2019, 12, 1665.

17. T.-H. Fang and W.-J. Chang, Applied Surface Science, 2005, 252, 1863-1869.

18. N. Kikuchi, E. Kusano, E. Kishio and A. Kinbara, Vacuum, 2002, 66, 365-371.

19. M. Ruske, G. Bräuer, J. Pistner, U. Pfäfflin and J. Szczyrbowski, Thin Solid Films, 1999, 351, 146-150.

20. J. B. Mooney and S. B. Radding, Annual review of materials science, 1982, 12, 81-101.

21. J. Sun, A. R. Pascoe, S. Meyer, Q. Wu, E. Della Gaspera, S. R. Raga, T. Zhang, A. Nattestad, U. Bach, Y.-B. Cheng and J. J. Jasieniak, Solar Energy, 2019, 188, 697-705.

22. H. Faber, B. Butz, C. Dieker, E. Spiecker and M. Halik, Advanced Functional Materials, 2013, 23, 2828-2834.

23. C. Barret and T. B. Massalski, Structure of Metals, Pergamon Press, Oxford, UK, 1980.

24. E. Della Gaspera, J. van Embden, A. S. R. Chesman, N. W. Duffy and J. J. Jasieniak, ACS Applied Materials \& Interfaces, 2014, 6, 22519-22526.

25. V. Consonni, G. Rey, H. Roussel, B. Doisneau, E. Blanquet and D. Bellet, Acta Materialia, 2013, 61, 22-31.

26. J. E. N. Swallow, B. A. D. Williamson, T. J. Whittles, M. Birkett, T. J. Featherstone, N. Peng, A. Abbott, M. Farnworth, K. J. Cheetham, P. Warren, D. O. Scanlon, V. R. Dhanak and T. D. Veal, Advanced Functional Materials, 2018, 28, 1701900.

27. G. Haacke, Journal of Applied Physics, 1976, 47, 4086-4089. 28. N. G. S. Energy, NSG TEC Datasheet, https://www.pilkington.com/en/global/products/product -categories/solar-energy/nsg-tec-for-solarapplications\#brochures).

29. D. Tatar and B. DÜZgüN, Pramana, 2012, 79, 137-150.

30. B.-R. Koo, D.-H. Oh, D.-H. Riu and H.-J. Ahn, ACS Applied Materials \& Interfaces, 2017, 9, 44584-44592.

31. R. Egdell, J. Rebane, T. Walker and D. Law, Physical Review B, 1999, 59, 1792.

32. P. Stefanov, G. Atanasova, E. Manolov, Z. Raicheva and V. Lazarova, Journal of Physics: Conference Series, 2008, 100, 082046.

33. F. Borgatti, J. A. Berger, D. Céolin, J. S. Zhou, J. J. Kas, M. Guzzo, C. F. McConville, F. Offi, G. Panaccione, A. Regoutz, D. J. Payne, J.-P. Rueff, O. Bierwagen, M. E. White, J. S. Speck, M. Gatti and R. G. Egdell, Physical Review B, 2018, 97, 155102.

34. I. ISO, 14577-4: Test method for metallic and non-metallic coatings, Technical report, International Organization for Standardization, 2004.

35. Q. Gao, H. Jiang, M. Li, P. Lu, X. Lai, X. Li, Y. Liu, C. Song and G. Han, Ceramics International, 2014, 40, 2557-2564.

36. W. C. Oliver and G. M. Pharr, Journal of Materials Research, 2011, 7, 1564-1583. 
37. R. A. Mirshams and R. M. Pothapragada, Acta Materialia, 2006, 54, 1123-1134.

38. K. Zeng, F. Zhu, J. Hu, L. Shen, K. Zhang and H. Gong, Thin Solid Films, 2003, 443, 60-65.

39. P. M. Nagy, P. Horváth, E. Kálmán and F. Kormos, Materials and Manufacturing Processes, 2005, 20, 115-122.

$40 . \quad$ H. S. Park, UC Berkeley, 2017.

41. S. Zhang, D. Sun, Y. Fu and H. Du, Surface and Coatings Technology, 2003, 167, 113-119.

42. T. Csanádi, M. Bl'anda, N. Q. Chinh, P. Hvizdoš and J. Dusza, Acta Materialia, 2015, 83, 397-407.

43. J.-W. Kim, K.-S. Kim, H.-J. Lee, H.-Y. Kim, Y.-B. Park and S.M. Hyun, Journal of the Microelectronics and Packaging Society, 2011, 18, 11-18.

44. J.-W. Kim, M.-H. Jeong and Y.-B. Park, Microelectronic Engineering, 2012, 89, 42-45. 